CORRECTION

\title{
Correction: MIF inhibition enhances pulmonary angiogenesis and lung development in congenital diaphragmatic hernia
}

Shahana Perveen ${ }^{1}$, Kamesh Ayasolla ${ }^{1}$, Nahla Zagloul ${ }^{1}$, Hardik Patel ${ }^{1}$, Kanta Ochani ${ }^{2}$, David Orner ${ }^{1}$, Helene Benveniste ${ }^{3}$, Michael Salerno ${ }^{4}$, Paul Vaska ${ }^{4,5}$, Zhang Zuo ${ }^{6}$, Yousef Alabed ${ }^{7}$, Mansoor Nasim ${ }^{8}$, Edmund J. Miller ${ }^{2}$ and Mohamed Ahmed ${ }^{1}$

Pediatric Research (2019) 85:735; https://doi.org/10.1038/s41390-019-0362-3

Correction to: Pediatric Research https://doi.org/10.1038/s41390019-0335-6, published online 13 February 2019
In the original version of this article, the name of the author "Kamesh Ayasolla" was incorrectly given as "Kamesh Ayyasola". This has now been corrected to "Kamesh Ayasolla" in both the PDF and HTML versions of the article.

\footnotetext{
'Division of Neonatal-Perinatal Medicine, Cohen Children's Medical Center, and Lilling Family Neonatal Research Laboratory, Feinstein Institute for Medical Research, Manhasset, NY, USA; ${ }^{2}$ Heart and Lung Research Unit, Feinstein Institute for Medical Research, Manhasset, NY, USA; ${ }^{3}$ Department of Anesthesiology, Yale School of Medicine, New Haven, CT,

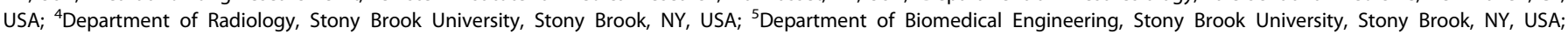
${ }^{6}$ Department of Chemistry, Stony Brook University, Stony Brook, NY, USA; ${ }^{7}$ Department of Medicinal Chemistry, Center for Molecular Innovation, Manhasset, NY, USA and ${ }^{8}$ Department of Pathology, Northwell Health, New Hyde Park, NY, USA

Correspondence: Shahana Perveen (sperveen1@northwell.edu)
}

Published online: 6 March 2019 